\title{
Quelques remarques pratiques pour les utilisateurs de EGS4 sous DOS 6.2
}

\author{
E. DUVERGER*, R. ISABEY*, M. HUGHET**, L. MAKOVICKA*
}

\begin{abstract}
(Manuscrit reçu le 15 mai 1996, révisé le 15 janvier 1997, accepté le 10 février 1997)

RÉSUMÉ Le code de calcul EGS4 développé au SLAC sous l'égide de Nelson et al. (1985) est particulièrement performant. Nous nous intéressons dans ce travail à l'installation du logiciel sous DOS 6.2 et Windows et à l'identification de quelques erreurs.

ABSTRACT The possibility to install EGS4, developped originally by Nelson et al. (1985), for Monte Carlo gamma - electron simulations under DOS 6.2 \& Windows and some errors identification in different mortran programs are presented in this note.
\end{abstract}

\section{Introduction}

Les utilisateurs du code EGS4 sont de plus en plus nombreux car c'est un outil très efficace pour la simulation des interactions des électrons et des gamma avec la matière (Nahum et al., 1988 ; Isabey et al., 1995...). Malheureusement, ils sont souvent confrontés, après avoir obtenu une des versions de base (par exemple, celle qui est généreusement disponible auprès de la banque du NEA), aux problèmes de l'installation de ce code de Monte-Carlo. Nous proposons de fournir dans la suite de ce travail, une méthode d'installation de ce code pour les nouveaux utilisateurs. Par la même occasion, nous rappellerons la méthode à utiliser pour corriger les erreurs qui existent dans les versions pour compatibles PC. Dans cette procédure, nous définissons l'approche la plus linéaire possible.

Elle est bien entendu modulable en fonction de l'environnement de chaque ordinateur. Il est recommandé de commencer par installer la version F32 du Fortran de Microsoft en choisissant les options pour la faire fonctionner sous DOS et Windows. La documentation du logiciel fournit toutes les informations utiles pour effectuer cette étape. On doit effectuer cette double installation

\footnotetext{
* L.M.I.T/R.M.C, Université de Franche-Comté, Rue Engel Gros, BP 527, 90016 Belfort, France.

** C.2.M/I.R.C.O.M, Université de Limoges, 123 Avenue A. Thomas, 87000 Limoges, France.
} 
pour éliminer des codes d'erreurs intervenant à la compilation. Nous supposons également que les futurs utilisateurs possèdent la documentation et la disquette source fournies par le N.E.A.

\section{Modification de son espace d'environnement}

Il nous reste à traiter plus particulièrement le problème de l'installation de la version DOS de EGS4 fournie gracieusement par le N.E.A en notant qu'il est indispensable de modifier les fichiers «Config.sys » et « Autoexec.bat » sur son ordinateur. Ces modifications sont essentielles pour pouvoir travailler sans problème sous DOS ou Windows. Nous envisagerons l'installation de EGS4 sur un ordinateur ne possédant qu'un seul disque dur.

Dans le fichier « Config.sys », il faut augmenter la taille du Buffer à 40 et le nombre maximal de fichiers (files $=80$ ) pouvant être ouverts afin de ne pas rencontrer de problème dans l'espace d'environnement. La compilation d'un fichier avec le F32 de Microsoft se fait alors sans aucune difficulté sous DOS.

Dans le fichier « autoexec.bat », il faut spécifier les chemins d'accès au répertoire qui contient le programme EGS4. Dans notre exemple, nous avons pris le répertoire mtegs

\section{C:IMTEGS ;C:IMTEGSIEGS4 ;C:IMTEGSIMORTRAN3}

Bien entendu, la moindre modification des fichiers «Config.sys » et "Autoexec.bat » devient effective en faisant redémarrer l'ordinateur. En cas de problème d'allocation mémoire, il faut utiliser la commande « memmaker » pour résoudre cette difficulté sous DOS.

Remarque : l'existence de plusieurs disques durs sur un ordinateur n'entrave en rien le fonctionnement de EGS4 et du Fortran F32. Il suffit de modifier les précédents fichiers en remplaçant le répertoire racine $\mathrm{C}$ par le nouveau nom du répertoire racine : par exemple D.

\section{Installation de EGS4}

Pour copier les fichiers de EGS4 sur son disque dur, il suffit de suivre la procédure éditée par le N.E.A et contenue sur la disquette dans le fichier « NeaRead.me ».

On lance ensuite la commande NEAINS C: (indique le lecteur cible sur lequel on veut faire l'installation). Un répertoire CCC0331.03 est créé sur le lecteur C. On passe ensuite dans le répertoire CCC0331.03 puis EGS4 et on effectue la commande suivante :

$$
\text { XCopy *.* C:I MTEGS } / / \mathrm{s}
$$


On accepte la création d'un répertoire MTEGS. Il nous reste à détruire le répertoire CCC0331.03 dont on n'a plus besoin. Sous MTEGS, on rentre dans le répertoire MORTRAN3 et on modifie tous les fichiers Batch à l'aide de l'éditeur DOS pour que les chemins logiques soient conformes avec notre environnement. Ainsi, en travaillant sur un disque dur $\mathrm{C}$, on transforme la commande :

\section{set EGS4_home=C:IEGS en set EGS4_home=C:IMTEGS}

L'ordinateur saura ainsi qu'il doit effectuer toutes les opérations définies dans les fichiers batch en les appliquant aux fichiers présents sur le disque dur $\mathrm{C}$ dans le répertoire MTEGS.

Il faut également supprimer les lignes suivantes dans « Makemor3.bat » :

- if \%debug \%==false 77713 mortran3.for $/ \mathrm{H} / \mathrm{NB} / \mathrm{NW} / \mathrm{NS} / \mathrm{NL} / \mathrm{NI} / \mathrm{NX}>$ mortran3.log

- if \%debug \%==true f7713 mortran3.for $/ \mathrm{H} / \mathrm{B} / \mathrm{W} / \mathrm{S} / \mathrm{L} / \mathrm{I} / \mathrm{X} \gg$ mortran3.log

pour les remplacer par :

- if \%debug\%==false FL32/G4/0x/Zi mortran3.for $>$ mortran3.log

- if \%debug\%==true FL32/G4/0x/Zi mortran3.for $>$ mortran3.log

Et on supprime définitivement les lignes :

- if exist mortran3.log del mortran3.log

- if exist mortran3.Ist del mortran3.Ist

- if exist mortran3.exe del mortran3.exe

- if exist mortran3.xmp del mortran3.xmp

- 386link mortran3.obj > mortran3.log

On lance ensuite l'exécution de « Rawtohex.bat » afin de traduire les règles de compilation en hexadécimal. Si on a suivi toutes les étapes, on ne doit voir apparaître aucun message d'erreur. Il reste à ouvrir le fichier obtenu « Mortran3.dat », à effacer la première ligne en veillant à ne laisser aucun blanc, à sauver et à sortir (utiliser la combinaison de touche sur le clavier « del $\mathrm{Y} »$ ). On n'a plus à revenir dans le répertoire MORTRAN3. On poursuit l'installation par un passage dans le répertoire PEGS4 qui permet de déterminer les sections efficaces. On doit dans un premier temps éditer les fichiers batch de façon à modifier les arborescences en fonction de son environnement de travail :

\section{C:IEGS en C:IMTEGS}

On veille à remplacer dans le fichier « Makepegs.bat »:

- if not «\%1»==«debug» $\mathbf{f} 7713$ pegs 4 .for $>$ fortran.log

- if $« \% 1 »==\ll$ debug» 77713 pegs 4. for $/ H / B / W / S / L / I / X>$ fortran.log 
par :

- if not «\%1»==«debug» FL32/G4/0x/Zi pegs4.for > fortran.log

- if $« \% 1 »==«$ debug» $F L 32 / G 4 / 0 x / Z i$ pegs4.for $>$ fortran.log

On profite également de l'occasion pour éliminer les lignes suivantes :

- rem NEA-DB if exist pegs4.exp del pegs4.exp

- if exist pegs4.exe del pegs4.exe

- if exist pegs4.xmp del pegs4.xmp

- if exist link.log del link.log

- rem NEA-DB 132 pegs4.obj $/ \mathrm{M}$; > link.log

- 386link pegs4.obj > link.log

\section{Corrections des erreurs dans PEGS4}

Il nous reste maintenant à traiter les erreurs présentes dans le module

" Pegs4.mor ». Il faut copier les lignes suivantes avant de les effacer :

- DIMENSION STDAT1(6,20),STDAT2(6,20),STDAT3(6,20),STDAT4(6,13);

- EQUIVALENCE (STDATA(1,1),STDAT1(1,1));

- EQUIVALENCE (STDATA(1,21),STDAT2(1,1));

- EQUIVALENCE (STDATA(1,41),STDAT3(1,1));

- EQUIVALENCE (STDATA(1,61),STDAT4(1,1));

et les coller en-dessous de :

- \$TYPE MEDTB1(24,20),MEDTB2(24,20),MEDTB3(24,20),MEDTB4(24,13);

- EQUIVALENCE (MEDTBL(1,1),MEDTB1(1,1));

- EQUTVALENCE (MEDTBL(1,21),MEDTB2(1,1));

- EQUIVALENCE (MEDTBL(1,41),MEDTB3(1,1));

- EQUIVALENCE (MEDTBL(1,61),MEDTB4(1,1));

Dans le même fichier, il faut modifier la subroutine HPLT1 en supprimant la ligne :

- INTEGER ICAP (12), NH (200);

et en la remplaçant par :

- INTEGER NH (200);

On transforme également la ligne :

- TYPE L(100),CM,CR,CD,CBL ;

par :

- \$TYPE L(100),CM,CR,CD,CBL, ICAP (12); 
On supprime la ligne :

- CALL UNDER0 (. True.) ;

Elle ne fonctionne pas avec le Fortran Microsoft. On peut obtenir de plus amples informations en lisant la ligne 558.

On peut alors lancer sans aucune appréhension la transformation de «Pegs4.mor " en un fichier exécutable. On continue l'installation de EGS4 en modifiant les fichiers «Pegs4pepr.dat » et « Pegs4form.dat ». Il faut changer \&END en $/ \&$ END à la fin des fichiers sinon «Pegs4.exe » ne peut créer les fichiers contenant les sections efficaces.

Il faut également modifier tous les fichiers de la forme «*.inp » fournis avec le logiciel car ils ne peuvent fonctionner dans leur format actuel. Il faut à nouveau remplacer \&END par $/ \&$ END Pour terminer notre tour d'horizon dans le répertoire PEGS4, il faut modifier la fin de « Pegs4B.bat » en effaçant les lignes suivantes:

- rem The following three lines were added at the NEA-DB

- if exist \%1.bak del \%1.bak

- if exist user.dat del user.dat

- if exist user.inp del user.inp

et les remplacer par :

- move \%1. DAT C:IMTEGSIPEGS4IDAT

On vient d'en finir avec les modifications du répertoire PEGS4. On teste la qualité des modifications en lançant la commande :

\section{Pegs4brun P4iH202}

On doit créer un fichier « P4iH202.dat » dans le répertoire DAT. En cas d'erreur, il faut revoir les modifications précédentes.

\section{Correction des erreurs dans EGS4}

On passe dans le répertoire EGS4 puis on crée le répertoire FIRAM. Il nous permettra de différencier notre espace de travail des programmes mortran existants. Il faut à nouveau éditer les fichiers Batch présents dans le répertoire EGS4 et les modifier en fonction de notre environnement de travail :

\section{C:IEGS en C:IMTEGS}

On effectue le plus grand nombre de modifications dans le fichier « Egs4bcom.bat ». On commence par supprimer les lignes suivantes dans ce fichier : 
- if exist Fortran.log del fortran.log

- rem NEA-DB if exist \%1.exp del \%1.exp

- if exist \%1.exe del \%1.exe

- 386link \%1.obj > link.log

et on remplace :

- if \%debug $\%==$ false $\mathrm{f} 7713 \% 1$ for $/ \mathrm{H} / \mathrm{NB} / \mathrm{NW} / \mathrm{NS} / \mathrm{NL} / \mathrm{NI} / \mathrm{NX}>$ fortran.log

- if \%debug \%==true f7713 \%1.for $/ \mathrm{H} / \mathrm{B} / \mathrm{W} / \mathrm{S} / \mathrm{L} / \mathrm{I} / \mathrm{X} \gg$ fortran.log

par :

- if \%debug $\%==$ false fl32 $/ \mathrm{G} 4 / 0 \mathrm{x} / \mathrm{Zi} \% 1$. for $>>$ fortran. $\log$

- if \%debug $\%==$ true $\mathrm{fl} 32 / \mathrm{G} 4 / 0 \mathrm{X} / \mathrm{Zi} \% 1$. for $>>$ fortran.Log

On peut noter que le code EGS4 fourni par le N.E.A comprend une ébauche du code de calcul PRESTA (Bielajew et al., 1987) dans « Nrc4macp.mor ». Malheureusement, ce code a été développé à l'origine pour des symétries cylindriques. Il est donc préférable de modifier le fichier «Stdconf.bat » pour éliminer ce problème en remplaçant les lignes :

- copy \$mortjob.mor + c:lmtegslegs4lnrc4macp.mor \$mortjob.mor > \%1.log

- copy \$mortjob.mor + c:Imtegslegs4lnrccauxp.mor \$mortjob.mor > \%1.log par :

- copy \$mortjob.mor + c:lmtegslegs4lnrcc4mac.mor \$mortjob.mor > \%1.log - copy \$mortjob.mor + c:Imtegslegs4lnrccaux.mor \$mortjob.mor > \%1.log

Nous terminerons cette installation en ajoutant dans le fichier «Egs4mac.mor » la ligne :

\section{REPLACE \{CALL EXIT\} WITH $\{$ STOP\} ;}

On peut tester notre installation en compilant le fichier « tutor1.mor " présent dans le répertoire TUTOR. Pour cela, on le copie dans le répertoire FIRAM et on exécute egs4bcom. Mais attention, tous les fichiers mortran que l'on développe doivent contenir deux lignes supplémentaires placées au-dessus de :

\section{REPLACE \{\$MXMED\} WITH $\{1\}$;}

Ce sont les lignes :

- REPLACE [\$MXDATA\} WITH $\{1\}$;

- REPLACE \{\$STAT\} WITH $\{1\}$; cation.

Vous pouvez consulter le répertoire Appendix pour en connaître la signifi- 
L'utilisation de EGS4 en environnement multitâche est très simple. On lance une session Windows puis, on clique sur l'icône commandes MS-DOS. Nous sommes alors dans un environnement multitâche. On peut lancer un programme EGS4 tout en écrivant un article sous Windows etc.

\section{Conclusion}

L'installation de EGS4 sous DOS a été rendue la plus conviviale possible. Nous espérons de cette manière que les personnes tentées par son utilisation seront de plus en plus nombreuses.

Le bon fonctionnement de ces modifications a été confirmé par l'expérience (Isabey et al., 1996) et par des intercomparaisons des résultats obtenus par d'autres laboratoires français (Magnac et al., 1996).

Remerciements : Nous remercions particulièrement la banque de données de l'A.E.N-O.C.DE, les auteurs du code ainsi que l'association F.I.RAM pour son aimable aide et sa disponibilité.

\section{RÉFÉRENCES}

Bielajew A.F. and Rogers DW.O. (1987) Nuclear Instrum. Methods, B18, 165-181.

Isabey R., Duverger E., Makovicka L., Klein D., Chambaudet A. (1995) Modelisation of Gamma Germanium Detector response in different environmental conditions, Acta Polytechnica, 35 (4), 88-92.

Isabey R. , Duverger E., Makovicka L., Klein D., Chambaudet A. (1996) Optimisation des différents systèmes de détection sensibles aux rayonnements gamma, Journées S.F.R.P Codes de calcul en radioprotection, CEA-Saclay, 20-21 novembre 1996.

Magnac Y. , Nadai J.P. , Barthe J., Paul D. , Duverger E. , Makovicka L. , Gouriou J. (1996) Intercomparaison des divers codes de simulation au sein de la F.I.RAM, Journées RADECS 96, Nîmes, 9-10 septembre 1996.

Nahum A.E. (1988) Simulation of Dosimeter Response and Interface effects (Plenum Publishing Corporation) pp. 523-547.

Nelson W.R., H. Hirayama and Rogers DW.O. (1985) The EGS4 code system, Standford Linear Accelerator Center Report, SLAC-265. 